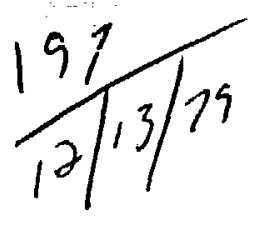

\title{
MASTER
}

\section{FALLOUT MODEL FOR SYSTEM STUDIES}

\author{
T. F. Harvey \\ F. J. D. Serduke
}

October 24, 1979

Work performed under the auspices of the U.S. Department of

Energy by the UCLLL under contract number W-7405-ENG-48.

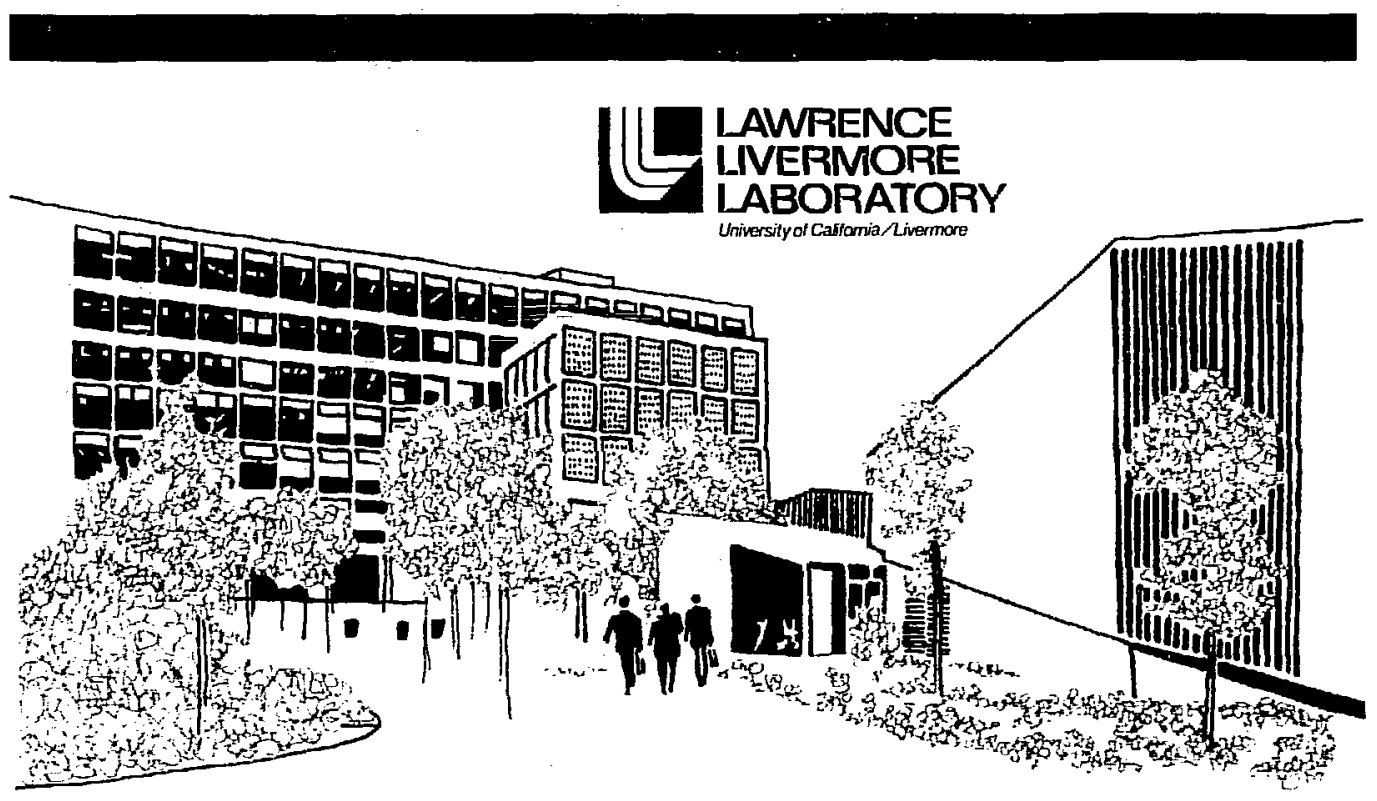




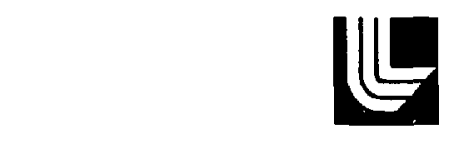

LAWRENCE LIVERMORE LABORATORY

University of Caftomia Livermore, Calitomia 94550

\title{
UCRL-52858 \\ FALLOUT MODEL FOR SYSTEM STUDIES
}

\author{
T. F. Harvey \\ F. J. D. Serduke
}

Manuscript date: October 24, 1979 


\section{CONTENTS}

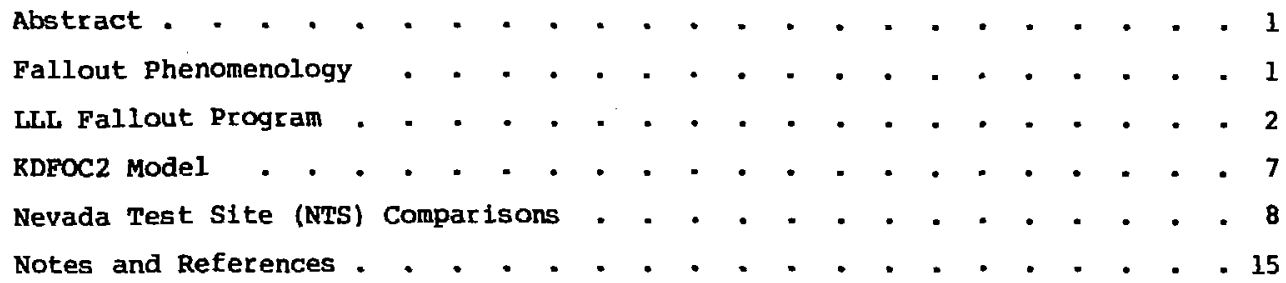




\section{FALLOUT MODEL FOR SYSTEM STUDIES}

ABSTRACT

A versatile fallout model has been developed to assess complex civil defense and military effect issues. Large technical and scenario uncertainties require a fast, adaptable, time-dependent model to obtain technically defensible fallout results in complex demographic scenarios.

The RDFOC2 capability, coupled with other data bases available at Lawrence Livermore Laboratory (LLL), provides the essential tools to ( 1 ) consider tradeoffs between various plans and features in aifferent nuclear scenarios and (2) estimate the technical uncertainties in the predictions.

All available data have been used to validate the model. In many ways, the capability is unmatched in its abillty to predict fallout hazards to a society.

\section{FALLOUT PHENOMENOLOGY}

During a nuclear detonation, everything in the device and immediately surrounding it vaporizes at extremely high temperatures, forming a fireball. In all but deeply buried bursts, this fireball rushes upward, cooling by radiation and then by expansion and entrainment of air and creating a strong, turbulent updraft. The center of the fireball is hottest. So it rises fastest, quickly converting the rising bubble into a smoke ring or toroid.

The vapors inside the nuclear fireball are intensely radioactive. This radioactivity comes from two main sources:

- Fission fragments from the detonation.

- Device debris and surrounding material that have been activated by absorbing the neutrons released during the detonation.

Many effects influence the proportion of these two sorts of radioactivity in the fallout. These effects include the fusion/fission ratio ("cleanness") of the device, the external neutron fluxes, and the kind and amount of surrounding material (the "environment" of the detonation, i.e., airburst, surface, or buried).

As the fireball cools, the material in it condenses into solid particles that grow as more material condenses on their surfaces. How large they grow 
depends in part on the density of the fireball, i.e., how much material it contains per unit volume. A free-air burst, one whose fireball never reaches the ground and which therefore involves nearly nothing condensable outside the device, will produce tiny particles (a few tenths of a micrometre). If the fireball touches the ground, as in a surface burst, it will vaporize some of the nearby soil and produce particles hundreds and thousands of times bigger. Also, the updraft from a near-surface, surface, or shallowly buried burst sweeps up dust, sand, and gravel into the rising debris cloud where each of these particles receives a radioactive coating.

Deeply buried bursts produce a cavity lined with molten radioactive soil and rock. Radioactive glass-like particles develop when the liner bursts at venting. Additional radioactive particles form when the rising debris cloud percolates through and condenses on the lofted soil. Much of this lofted soil falls back to the surface within several tens of seconds, creating a base surge that moves radially outward from ground zero. This base surge is capable of transporting signiflcant amounts of radioactivity.

In any burst that forms a rising debris cloud, the largest and heaviest particles detrain first, followed by smaller and smaller particles. For a given set of conditions, the smallest fallout particles rise highest and stay up for the longest time. The winds determine how far and in what direction they will travel in that time. Most of the particles from a free-air burst are so tiny that unless rain brings them down they will stay up for days, weeks, or even months. ${ }^{1}$ Hence the local fallout (within a few miles of ground zero) from such a burst is relatively insignificant, even from an allfission device.

The fallout from a surface or burled burst is in a different class. Many of the particles are large enough (hundreds of micrometres) to return to earth within a few hours. Some of them arrive within a few tens of seconds. Hence they are concentrated Into a much smaller area. Under such conditions the local fallout radiation may be intense even when the device detonated is relatively clean.

LLL FALLOUT PROGRAM

A 1975 LLL fallout code comparison study ${ }^{2}$ showed that existing fallout prediction techniques agreed to within a factor of five for the hazard from an 
all-fission surface burst (Fig. 1). This level of agreement deteriorated markedly when these prediction schemes were used to assess the same hazard level from a clean device (Fig. 2).

With the exception of the Defense Nuclear Agency's (DNA's) DELFIC, ${ }^{3}$ which gives credible results but is too cumbersome for assessment use, fallout codes generally begin by assuming a stabilized debris cloud that is eventually deposited on the ground by winds and gravitational settling. This is also true of the original KDFOC, which was primarily concerned with the relatively low-level, wide-area fallout hazard from Plowshare-type detonations. The fallout that develops before cloud stabilization is almost always overlooked in these formulations. This prestabilization fallout is of great interest in clean-weapon fallout assessment.

Furthermore, most assessment codes lacked a combined buried- and surfaceburst capability. Without specific attention to the transition from surfaceto shallow-burial detonations, these codes could give faulty assessments for shallow-burst munitions.

Our objective was to develop a rapid, relatively inexpensive fallout code that could completely model

- All-fission as well as clean weapons.

- Militarily significant radiation levels.

- Surface, shallow, and deeply burien detonations.

- Tactical yield ranges.

The model had to be continuous in all physical quantities, both as a function of depth of burial and of variations in the specified winds.

Two choices were available to us. We could modify DELFIC to make it more amenable to assessment studies and include a buried-burst capability. Or we could pursue the more phenomenological approach of the original kDFOC, improving it to account for cloud-rise fallout. We opted for the latter. A new approach to cloud-rise simulation was warranted, one that provided enough detail to reproduce the sallent features of observed fallout patterns without wasting effort in arbitrary physical detall.

The empirical establishment of initial conditions is a time-reversal process (Fig. 3) in which the fallout particles are projected from their actual landing points backward in time to $\mathrm{H}$ hour. This produces a taperad, effective stem cloud over ground zero. To this cloud, we could assign in appropriate distribution of debris with respect to altitude necessary to account for the 


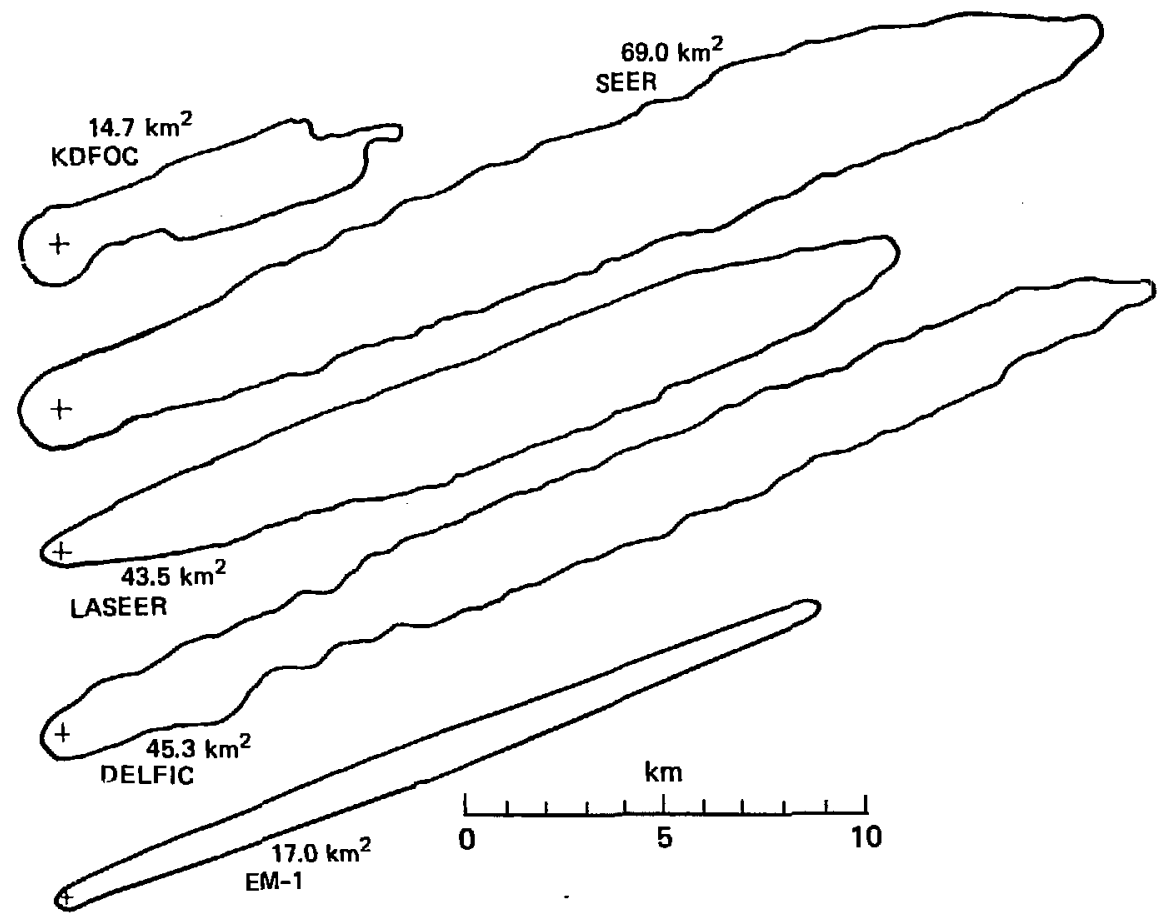

FIG. 1. The different fallout patterns predicted in 1975 by five fallout models for the same 8-TJ (2-kt) fission surface burst. KDFOC is the early Lawrence Livermore Laboratory code; SEER was developed by Stanford Research Institute, LASEER by Los Alamos Scientific Laboratory, DELFIC and EM-1 by the Defense Nuclear Agency. EM-1 is a graphical technique, the others are numerical models. In this example, we used low-shear, moderate-speed winds. The areas inside the various contours agree within a factor of five--there is a consensus among these codes for surface-burst, fission weapons. The radiation level inside the isodose contour is similar to that associated by the U.S. Army with emergency risk to exposed personnel. 


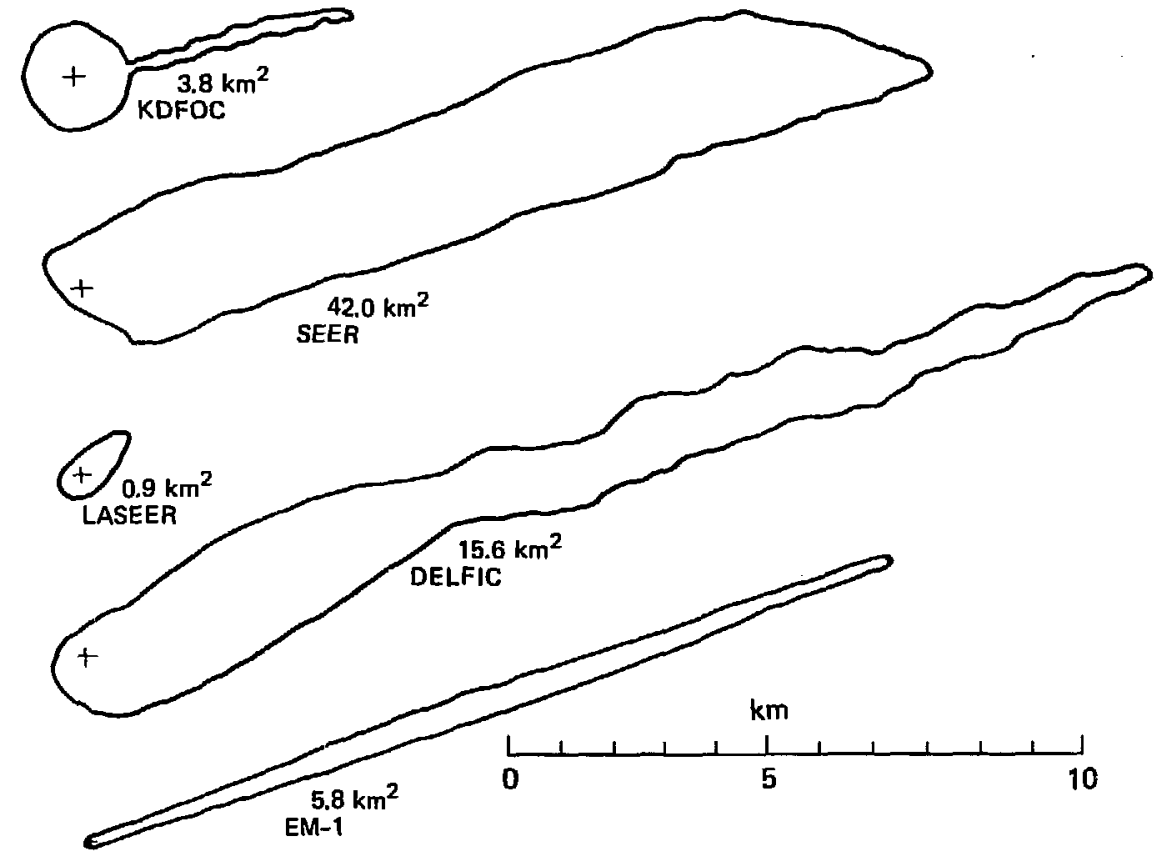

FIG. 2. Fallout patterns predicted in 1975 by the same five fallout models as in Fig. 1 for another surface burst at a different yiela for a clean weapon. Winds and exposure levels remain the same as in Fig. 1 . There is a wide range of disagreement_-_almost a factor of $\mathbf{5 0}$. 

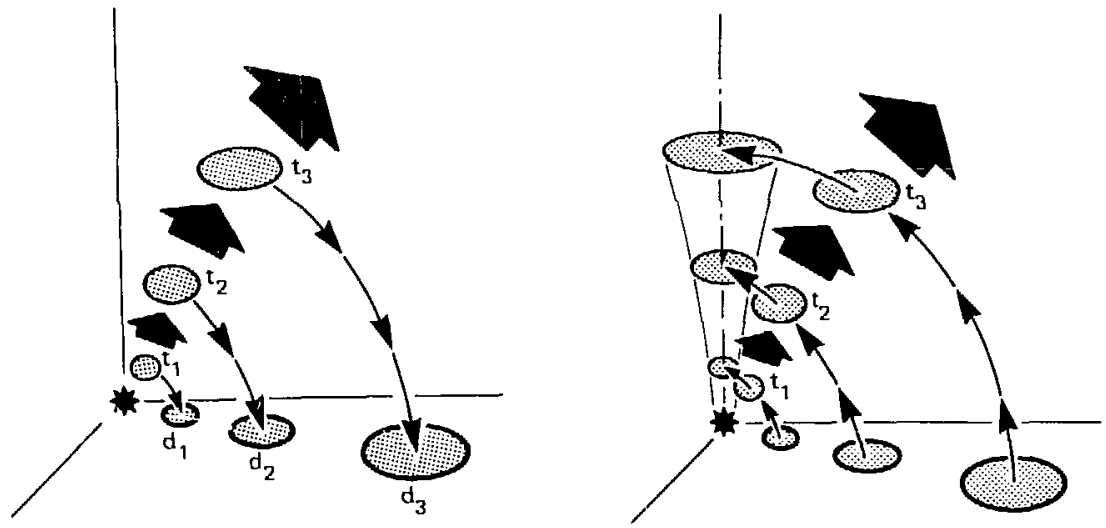

FIG. 3. A conceptual schematic of the KDFOC2 cloud-rise simulation. In the actual fallout process, radioactive products condense on particles with enough mass to detrain at times $t_{1}, t_{2}$, and $t_{3}$ from the rising toroidal debris mass. Larger fallout particles detrain earlier, represented schematically by $t_{1}$, and deposit closer to ground zero $\left(d_{1}\right)$. Smaller particles detrain at later times and deposit at greater distances $\left(d_{2}, d_{3}\right.$, etc.). From a knowledge of the winds and the settling speeds of the radioactive particles, Wh can project the particles backward in time (right) to a tapered, effective stem cloud above ground zero. With this approximation, we can then model the cloud-rise episode by choosing a simple but appropriate altitude distribution of radioactive debris. We then determine the few parameters in this simple model using the Nevada Test Site data.

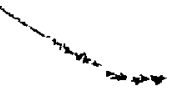


high radiation levels developed during the cloud-rise phase. The success of such a procedure would be tested by the model's overall fit to the data. We aimed to achieve agreement within a factor of several for both clean and allfission detonations.

KDFOC2 MODEL

The KDFOC2 model ${ }^{4}$ establishes, at h-hour, the dimensions of the initial debris configuration as well as the distributions of activity with respect to particle size and altitude. As time proceeds, suitably chosen parcels of debris follow trajectories defined by the winds, turbulent diffusion, and gravitational settling. When all the parcels hit the ground, their activity contributions are summed to yield an overall fallout pattern.

The required input for KDFOC2 has been kept to a minimum to make the code easy to apply. The only requirement is specification of a yield. All other parameters, if not otherwise specifled, are assigned default values that specify an all-fission surface burst with moderate-speed, low-shear winds. The standard output consists of a graphical hardcopy display of the fallout patterns at the dose levels requested. The display also lists the downwind distance and areal coverage of each of the contours.

Notable Features of KDFuC2 include

- Simulated cloud-rise. KDFOC2 explicitly considers the prestabilization Eallout.

- Efficient algorithms that lead to a fast-running code yielding statistically smooth fallout patterns in which all the radioactivity is conserved.

- Various dose and dose-rate options. These options include $\mathrm{H}+1$ dose rates for comparison with measured fallout patterns, infinite whole-body doses, finite whole-body doses, and dose rates at any time after detonation.

- Multiburst scenario assessment capability. A one-thousand 4-PJ (1-Mt) weapon attack was modeled for a civil defense study. 'The entire calcuiation took about a minute of $\mathrm{CDC}-7600$ central processing unit time.

- Time-varying winds.

- Active programming support. We have a continuing commitment to maintain KDFOC2. 
The DNA's recent fallout-code comparison study ${ }^{5}$ covered the only 10 well-documented cases of close-1n fallout from land-surface and buried bucsts detonated by the U.S. The DNA selected 38 of the isodose contours reported for the 10 test events. Table 1 lists these 10 events, together with thelr depths of burial and the selected isodose contours.

TABLE 1. NrS shots used for comparing fallout models.

\begin{tabular}{|c|c|c|}
\hline Event & $\begin{array}{c}\text { Depth of } \\
\text { burial } \\
\text { (m) }\end{array}$ & $\begin{array}{l}\text { Dose rates } \\
(\mathrm{Gy} / \mathrm{h} \text { at } \mathrm{H}+1 \mathrm{~h})^{6}\end{array}$ \\
\hline Small Boy & -3 & $5,2,1,0.50,0.20$ \\
\hline Jangle Sugar & -1 & $5,3,1,0.35$ \\
\hline Little Feller II & -1 & $2,1,0.5,0.2$ \\
\hline Johnnie Boy & 1 & $1,0.5,0.1$ \\
\hline Jangle Uncle & 5 & $5,2,1$ \\
\hline Teapot Ess & 20 & $5,1,0.5,0.05$ \\
\hline Danny Boy & 34 & $1,0.5,0.1,0.05$ \\
\hline Cabriolet & 52 & $1,0.1,0.01$ \\
\hline Palanquin & 85 & $5,1,0.5,0.05$ \\
\hline Schooner & 108 & $1,0.2,0.1,0.01$ \\
\hline
\end{tabular}


The winds used in the comparison study were from wind soundings taken in the test area at shot time. These soundings were usually near ground zero. A sounding reports a horizontal wind speed and its direction at each of about 10 altitudes ranging from surface level to well above the eventual top of the debris cloud. These wind soundings do not report any vertical component of the true winds nor do they reflect the terrain features or time variations of true winds. For purposes of fallout modeling, however, we must assume that the single reported B-hour sounding is characteristic of the winds of all times and locations of interest.

Despite vagaries of the winds, the correlation between the reported and KDEOC2-calculated isodose contours is impressive. A compariscn of the downwind extents of the measured and computed contours is graphically shown in Fig. 4. Each dot represents the downwind distance of a single contour; distance from the central diagonal line is a measure of the degree of disagreement. Points lying within the gray band represent agreement within a factor of two between the model and observation; 35 of 38 comparison points fall within this corridor. The measured and calculated areas for the same set of 38 isodose contours are compared in Fig. 5. Again only two of the points representing isodose areas are outside the colored factor-of-two band.

Figure 6 compares $\mathrm{H}+\mathrm{I}$ isodose contours computed by KDFOC2 for the Small Boy and Cabriolet Events with those observed at NTS. Small Boy was a near surface shot; Cabriolet was a buried plowshare shot. On the left are contours of 5, 2, 1, 0.5, and $0.2 \mathrm{~Gy} / \mathrm{h}$ for the Small Boy Event: on the right are contours of $1,0.1$, and $0.01 \mathrm{~Gy} / \mathrm{h}$ for Cabriolet.

The Rowland-Thompson overlap method, ${ }^{7}$ yet another way of measuring agreement between two sets of contours, computes a figure of merit based on the areas common to the two sets of contours being compared. Specifically, it computes the ratio of the dose-rate-weighted sum of the common areas to the dose-rate-weighted sum of the larger areas, with 1008 indicating a perfect match. The recent DNA study used this Rowland-Thompson overlap as its primary base of comparison.

In Table 2, we summarize the Rowland-Thompson overlaps for each of the 10 nuclear events based on the fallout predictions of KDFOC2 and the 6 codes in the DNA study. Three of the 6 codes are designed to deal only with surface bursts. For each event, the best overlap is highlighted with brackets; only one is outside the KDFOC2 column. 


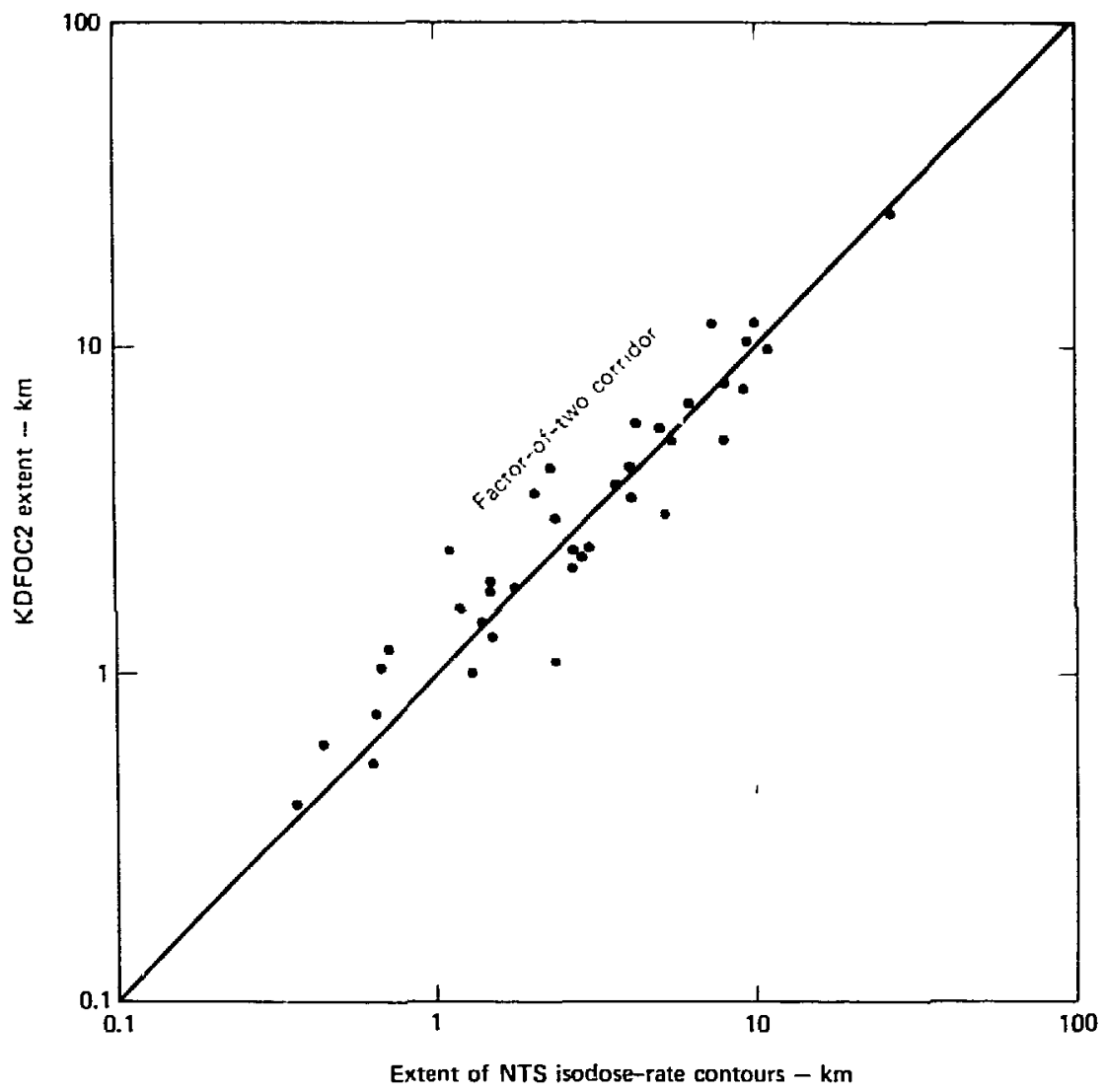

FIG. 4. Comparison between the measured and the calculated downwind extents of the 38 fallout contours listed in Table 1, with RDFOC2-calculated extents plotted on the ordinate and measured extents plotted on the abscissa. A result falling on the central diagonal line indicates exact agreement; a result falling within the gray band agrees within a factor of two. Only 2 of the 38 results are outside the. factor-of-two band; 708 of the results are within a factor of 1.4. Both measured and calcutated extents for the contours from the Little Feller II Event were multiplied by 10 to bring them onto the chart. 


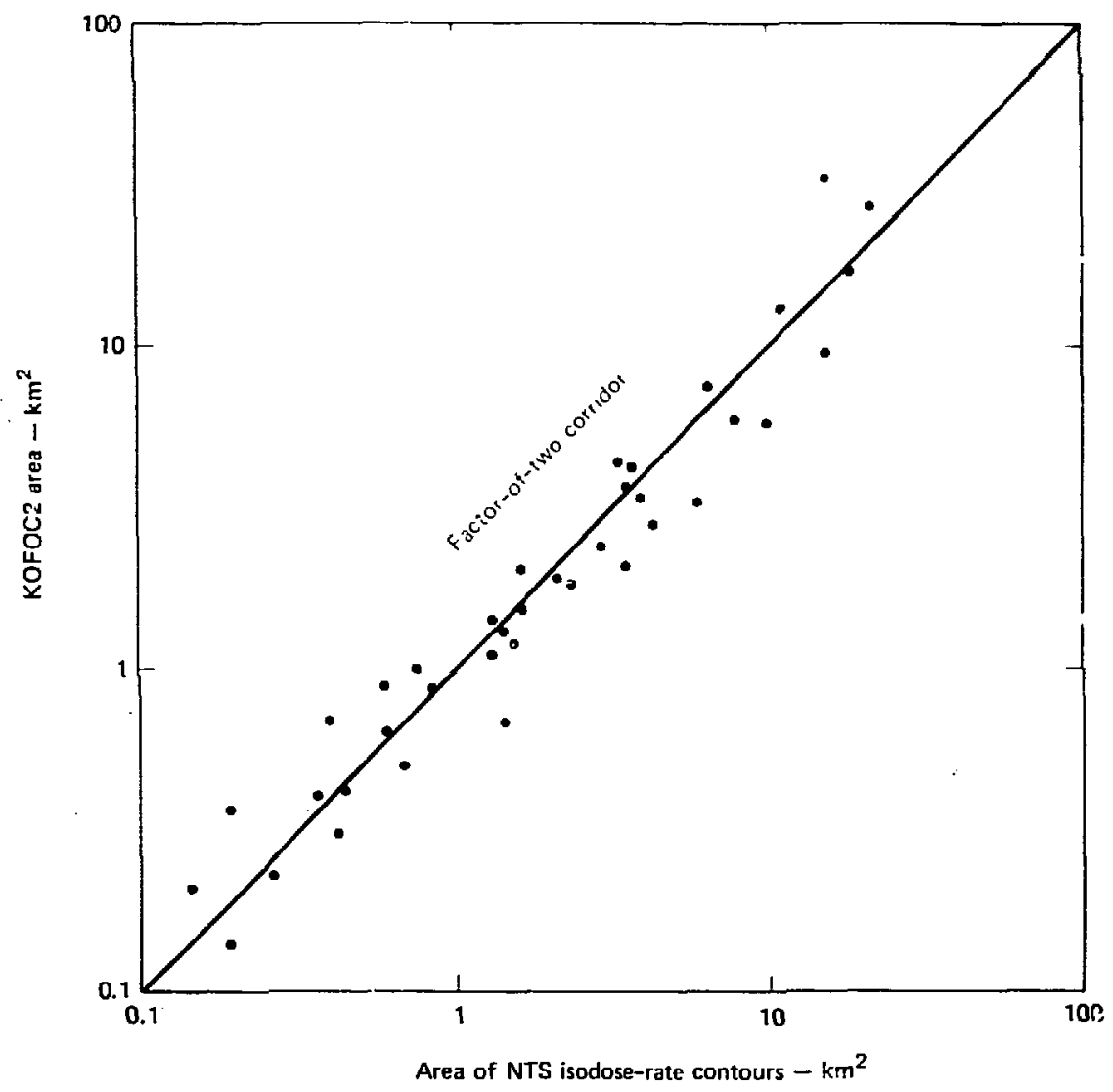

FIG. 5. Comparison of the measured and calculated area of the 38 fallout zontours listed in Table 1, with KDFOC2-calculated results plotted on the ordinate and measured areas plotted on the abscissa. As in Fig. 4, a result falling on the central diagonal line indicates exact agreement; a result falling within the gray band agrees within a factor of two. Again only two of the results (not the same as in Fig. 4) are outside the factor-of-two band; and again 708 of the results are within a factor of 1.4. Results from the Little Feller II Event were multiplied by a factor of 100 to bring them onto the chart. Schooner results were divided by 10 for the same reason. 


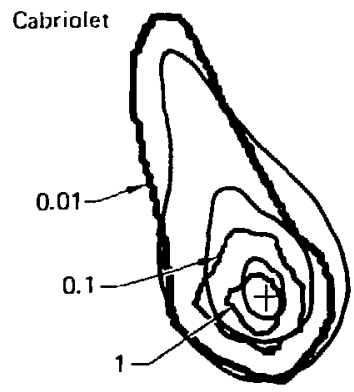

$2 \mathrm{~km}$

$1 \mathrm{~km}$

FIG. 6. Observed (solid) and computed (squiggly) fallout patterns for the Small Boy (left) and Cabriolet (right) Events. Small Boy was a nearly allfission device detonated at the surface. Cabriolet was a buried plowshare device. Dose rates are given in $\mathrm{Gy} / \mathrm{h}$ at $\mathrm{H}+1$ ( $1 \mathrm{~h}$ after detonation); $1 \mathrm{~Gy}=$ 100 rad. The KDFOC2 predictions match these data closely. This corroboration illustrates the utility of the code for a wide range of various physical parameters. 
TABLE 2. Rowland-Thompson overlaps (8) between measurements on 10 NTS and 1sodose contours calculated for these shots w1th KDFOC2 and 6 other popular fallout models. Results for codes other than KDPOC2 are taken from a draft version of the recent DNA fallout study. ${ }^{3}$ For depth of burlal information on each of these shots, see Table 1 . Best overlap for each event is indicated in brackets.

Numerical Model

\begin{tabular}{|c|c|c|c|c|c|c|c|}
\hline Event & KDFOC2 & PROFET & DELFIC & AUGER & KDFOC & LASEER & SEER \\
\hline Small Boy & $\{65\}$ & 41 & $16^{a}$ & 49 & 48 & 23 & 32 \\
\hline Jangle Sugar & 45 & 40 & 29 & 13 & 12 & 20 & $12^{b}$ \\
\hline Little Feller II & $\{44$ & 42 & 39 & 9 & 9 & 4 & $0^{c}$ \\
\hline Johnnle Boy & 48 & 45 & $\{53\}$ & 43 & 29 & 28 & 25 \\
\hline Jangle Uncle & 60 & - & 46 & 34 & 51 & 54 & -- \\
\hline Teapot Ess & $\{52$ & -- & -- & 27 & 27 & 35 & -- \\
\hline Danny Boy & 66 & - & - & 13 & 2 & 8 & - \\
\hline Cabriolet & $56\}$ & - & - & 1 & 1 & 7 & -- \\
\hline Palanquin & $\left\{39^{d}\right\}$ & -- & - & 36 & 26 & 36 & -- \\
\hline Schooner & $\{44\}$ & - & - & 35 & 37 & $14^{b}$ & -- \\
\hline Average & 51.9 & 42.0 & 36.6 & 26.0 & 24.2 & 22.9 & 17.3 \\
\hline
\end{tabular}

\footnotetext{
a An error was made in running DELFIC for this case; DELFIC has done significantly better for small Boy in the past.

${ }^{b}$ code predicted only two contours instead of the four measured.

c code predicted only one contour instead of the four measured.

d Rotating the wind by $17^{\circ}$ changes this overlap to 658 .
} 
By any measure, whether by areal coverage, downwind extent, or RowlandThompson overlap, RDFOC2 shows an unprecedented level of agreement with the reported data. Its speed, accuracy, and ease of application, taken together, make it a clearly superior fallout assessment code. 
1. Ralnout from air bursts is discussed in the Research Monthly, Lawrence L1vermore Laboratory, L1vermore, Cal1f., UCRL-50000-76-5 (May 1976), P. 7.

2. Our 1975 fallout code comparison study is descrlbed in the Research Monthly, Lawrence Livermore Laboratory, Livermore, Calif., UCRL-50000-75-7 (July 1975), P. 4.

3. DELFIC, a surface-burst model, is quite decalled in its capabilitieg. It accounts for cloud-rise by considering the motion of a cooling, buoyant bubble--a simplistlc model, but one that lends some credibility to the predicted close-in fallout patterns. However, it is generally agreed that DELFIC is too cumbersome for general use in fallout assessment. Also, DELFIC is unable to deal with buried bursts.

4. T. F. Harvey, F. J. D. Serduke, and A. C. Springer, KDFOC2, A NEW Fallout Assessment Capability, Lawrence Livermore Laboratory, Livermore, Callf., to be published.

5. H. G. Norment, Analysis and Comparison of Fallout Prediction Models, Defense Nuclear Agency, Washington, D.C., DNA 4569F (1977). This report analyzes elght fallout prediction models (not including RDFOC2); it compares their model structures and how we.i they agree with observed data.

6. The Gray (Gy) is an ST unit of radiation dose: $1 \mathrm{~Gy}=1 \mathrm{~J} / \mathrm{kg}=100$ rad.

7. R. H. Rowland and J. H. Thompson, A Method of Comparing Fallout Patterns, Defense Nuclear Agency, Washington, D.C., DNA 2919F (1972). 